OPEN ACCESS

Edited by:

Gaetano Ruocco,

Regina Montis Regalis Hospital, Italy

Reviewed by:

Alberto Aimo,

Sant'Anna School of Advanced

Studies, Italy

Kenichi Hongo,

Jikei University School of

Medicine, Japan

*Correspondence:

Sofia Morra

sofia.morra@erasme.ulb.ac.be

Lorenzo Pitisc

lorenzo.pitisci@ulb.be

tThese authors have contributed equally to this work

Specialty section:

This article was submitted to Heart Failure and Transplantation,

a section of the journal

Frontiers in Cardiovascular Medicine

Received: 06 September 2020

Accepted: 08 February 2021

Published: 08 March 2021

Citation:

Morra S, Pitisci L, Su F, Hossein A

Rabineau J, Racape J, Gorlier D,

Herpain A, Migeotte P-F, Creteur J

and van de Borne $P$ (2021)

Quantification of Cardiac Kinetic

Energy and Its Changes During

Transmural Myocardial Infarction

Assessed by Multi-Dimensional

Seismocardiography.

Front. Cardiovasc. Med. 8:603319.

doi: 10.3389/fcrm.2021.603319

\section{Quantification of Cardiac Kinetic Energy and Its Changes During Transmural Myocardial Infarction Assessed by Multi-Dimensional Seismocardiography}

\author{
Sofia Morra ${ }^{1 \star \dagger}$, Lorenzo Pitisci ${ }^{1,2 * \dagger}$, Fuhong Su ${ }^{2}$, Amin Hossein $^{3}$, Jérémy Rabineau ${ }^{3}$, \\ Judith Racape ${ }^{4}$, Damien Gorlier ${ }^{3}$, Antoine Herpain ${ }^{2,5}$, Pierre-François Migeotte ${ }^{3}$, \\ Jacques Creteur ${ }^{5}$ and Philippe van de Borne ${ }^{1}$
}

\begin{abstract}
${ }^{1}$ Department of Cardiology, Erasme Hospital, Université Libre de Bruxelles, Brussels, Belgium, ${ }^{2}$ Experimental Laboratory of Intensive Care, Erasme Hospital, Université Libre de Bruxelles, Brussels, Belgium, ${ }^{3}$ Laboratory of Physic and Physiology (LPHYS), Université Libre de Bruxelles, Brussels, Belgium, ${ }^{4}$ Research Center in Epidemiology, Biostatistics and Clinical Research, School of Public Health, Université Libre de Bruxelles (ULB), Brussels, Belgium, ${ }^{5}$ Department of Intensive Care, Erasme Hospital, Université Libre de Bruxelles, Brussels, Belgium
\end{abstract}

Introduction: Seismocardiography (SCG) records cardiac and blood-induced motions transmitted to the chest surface as vibratory phenomena. Evidences demonstrate that acute myocardial ischemia (AMI) profoundly affects the SCG signals. Multidimensional SCG records cardiac vibrations in linear and rotational dimensions, and scalar parameters of kinetic energy can be computed. We speculate that AMI and revascularization profoundly modify cardiac kinetic energy as recorded by SCG.

Methods: Under general anesthesia, 21 swine underwent 90 min of myocardial ischemia induced by percutaneous sub-occlusion of the proximal left anterior descending (LAD) coronary artery and subsequent revascularization. Invasive hemodynamic parameters were continuously recorded. SCG was recorded during baseline, immediately and 80 min after LAD sub-occlusion, and immediately and 60 min after LAD reperfusion. iK was automatically computed for each cardiac cycle $\left(i K^{C C}\right)$ in linear $\left(i K_{L i n}\right)$ and rotational $\left(i K_{R o t}\right)$ dimensions. $i K$ was calculated as well during systole and diastole ( $i K^{\text {Sys }}$ and $i K^{\text {Dia }}$, respectively). Echocardiography was performed at baseline and after revascularization, and the left ventricle ejection fraction (LVEF) along with regional left ventricle (LV) wall abnormalities were evaluated.

Results: Upon LAD sub-occlusion, $77 \%$ of STEMI and $24 \%$ of NSTEMI were observed. Compared to baseline, troponins increased from $13.0(6.5 ; 21.3) \mathrm{ng} / \mathrm{dll}$ to 170.5 (102.5; 475.0) $\mathrm{ng} / \mathrm{dl}$, and LVEF dropped from $65.0 \pm 0.0$ to $30.6 \pm 5.7 \%$ at the end of revascularization (both $p<0.0001$ ). Regional LV wall abnormalities were observed as follows: anterior MI, $17.6 \%$ (three out of 17); septal MI, 5.8\% (one out of 17); antero-septal MI, 47.1\% (eight out of 17); and infero-septal MI, 29.4\% (five out of 17). In the linear dimension, $i K_{\text {Lin }}^{C C}, i K_{\text {Lin }}^{\text {Sys }}$, and $i K_{\text {Lin }}^{D i a}$ dropped by 43,52 , and 53\%, respectively $(p<0.0001$, $p<0.0001$, and $p=0.03$, respectively) from baseline to the end of reperfusion. In the 
rotational dimension, $i K_{\text {Rot }}^{C C}$ and $i K_{\text {Rot }}^{\text {Sys }}$ dropped by 30 and $36 \%$, respectively $(p=0.0006$ and $p<0.0001$, respectively), but $i K_{R o t}^{D i a}$ did not change $(p=0.41)$. All the hemodynamic parameters, except the pulmonary artery pulse pressure, were significantly correlated with the parameters of $i K$, except for the diastolic component.

Conclusions: In this very context of experimental AMI with acute LV regional dysfunction and no concomitant AMI-related heart valve disease, linear and rotational iK parameters, in particular, systolic ones, provide reliable information on LV contractile dysfunction and its effects on the downstream circulation. Multidimensional SCG may provide information on the cardiac contractile status expressed in terms of $i \mathrm{~K}$ during $\mathrm{AMl}$ and reperfusion. This automatic system may empower health care providers and patients to remotely monitor cardiovascular status in the near future.

Keywords: seismocardiography, kinetic energy, acute myocardial infarction, animal model for acute coronary syndrome, cardiac monitoring

\section{INTRODUCTION}

Ballistocardiography (BCG) and seismocardiography (SCG) record the micro-vibrations produced rhythmically by velocities and accelerations of blood mass flowing across cardiac chambers and main vessels as a consequence of cardiac mass contraction, with micro-accelerometers and gyroscopes placed on the body surface (1-3). There is growing evidence that BCG and SCG may provide additional relevant information on cardiovascular status beyond those already acquired by means of universally accepted current diagnostic devices. Indeed BCG and SCG reliably estimate stroke volume (SV) and cardiac output (CO) $(2,4)$, myocardial contractility expressed as $\mathrm{d} P / \mathrm{d} t_{\max }$ in animal models (5), as well as the clinical status of heart failure patients (6).

As a result, this evidence fuels the curiosity of scientific and medical researchers who actively inquire on the potential of BCG and SCG signals to assess cardiovascular mechanical changes during acute myocardial infarction (AMI) (5, 7-9). Indeed the BCG and SCG signals profoundly change during AMI, and, according to previous studies, metrics secured from it enable the identification of an impairment of regional myocardial contraction due to acute ischemia with specificity of $80 \%$ (7) to $92 \%$ (9) and sensitivity of $89 \%$ (7) to $94 \%$ (9). When combined with the electrocardiogram (ECG), the SCG empowers the capability of detection of coronary artery disease during an exercise stress test, yielding a positive predictive value of $88 \%$ and a negative predictive value of $80 \%$ (10).

Recently, a multi-dimensional BCG combined with a multidimensional SCG, called kinocardiograph (KCG), has been introduced and, differently from many previous devices which record signals only in one dimension, the KCG records both three-dimensional (3D) linear acceleration and 3D angular velocity signals by means of linear and rotational channels $(2,11)$. Using specific algorithms, kinetic energy and its temporal integral $(i K)$ can be computed from the BCG and SCG waveforms as scalar parameters, both in a linear $\left(i K_{\text {Lin }}\right)$ and in a rotational $\left(i K_{\text {Rot }}\right)$ dimension $(2,12)$.

Three-axes linear micro-accelerometers have already been shown useful in the early detection of experimental AMI $(7-9,13,14)$. However, whether non-invasive accelerometers and gyroscopes recording signals in multiple (linear and rotational) dimensions can be affected by hemodynamic changes during acute myocardial infarction and reperfusion is not known. Recently, non-invasive techniques based on microaccelerometers and gyroscopes exploring rotational velocities and accelerations produced by heart contraction have been introduced (15-17): rotational velocities measured using noninvasive tri-axial gyroscopes provide information on several mechanical events occurring during a contractile cycle as compared to echocardiography (16). The rotational kinetic energy obtained from tri-axial gyroscopes can accurately identify the first and the second peak of the SCG (15). Rotational, rather than linear, kinetic energy accounts for about $70 \%$ of the total cardiac energy produced during a contractile cycle, and it significantly drops after prolonged cardiac deconditioning, mainly due to a decrease in the rotational twist of the LV (18). Measuring the rotational accelerations and kinetic energy may contribute to a more in-depth and global analysis of cardiac function seen through the windows of micro-accelerations since the rotational motion of the heart along its longitudinal axis is crucial in assuring its pumping function (19).

Using an animal model for AMI, the present investigation aims (1) to track modifications of linear and rotational $i \mathrm{~K}$ computed from the accelerations signals of non-invasive and multidimensional SCG during coronary artery sub-occlusion, (2) to follow these changes during the reperfusion period, and (3) to study the association of linear and rotational $i \mathrm{~K}$ with invasive hemodynamic parameters. The hypothesis tested is that experimental AMI and reperfusion profoundly alter multidimensional SCG signals and its derived scalar parameters.

\section{MATERIALS AND METHODS}

\section{Study Protocol}

The present study was approved by the Institutional Ethics Committee on Animal Welfare from the Faculty of Medicine of the Université Libre de Bruxelles (ULB, Brussels, Belgium) (acceptance number: 654N). Animal care and handling were in accordance with the National Institute of Health Guidelines. 
The procedure consisted in the proximal left anterior descending coronary artery (LAD) sub-occlusion by means of angioplasty semi-compliant balloon inflation for $90 \mathrm{~min}$, followed by deflation and subsequent reperfusion (RE) for 60 min. A 3-min-length SCG was recorded during the steady state (baseline, BSL) preceding the LAD sub-occlusion and then at different time points during sub-occlusion and reperfusion, specifically at the onset of $\mathrm{LAD}$ sub-occlusion $\left(\mathrm{AMI}_{\mathrm{t} 0}\right)$ at 80 minutes of $\mathrm{AMI}\left(\mathrm{AMI}_{\mathrm{t} 80}\right)$, at the onset of $\mathrm{RE}\left(\mathrm{RE}_{\mathrm{t} 0}\right)$, and at $60 \mathrm{~min}$ of $\mathrm{RE}\left(\mathrm{RE}_{\mathrm{t} 60}\right)$. Each record was remotely acquired with a tablet and sent via Bluetooth to the main server for further signal processing. To evaluate the amount of myocardial necrosis, the authors measured the serum troponin levels at the onset of LAD sub-occlusion and after revascularization: the difference between troponins measured $5 \mathrm{~h}$ after $\mathrm{RE}$ and troponins measured at the onset of LAD sub-occlusion was named Delta $(\Delta)$ troponin. Echocardiography was performed at baseline and after revascularization, and the left ventricle ejection fraction (LVEF) along with regional left ventricle (LV) wall abnormalities were evaluated by a trained operator.

\section{Animal Preparation and Experimental Procedure}

The animals have been put on fasting for $18 \mathrm{~h}$ before the experiment was started, with unrestricted access to water. Twenty-one 50-kg crossbreed Landrace/Large White adult swine, of either sex, were premedicated with intramuscular neck injection of $5 \mathrm{mg} / \mathrm{kg}$ azaperone and $1.5 \mathrm{mg} / \mathrm{kg}$ midazolam. A $14-\mathrm{G}$ peripheral venous line was placed into an ear vein to provide vascular access, and a 4.5-Fr arterial catheter (LeaderCath, Vygon, France) was inserted in the left common femoral artery for invasive arterial pressure monitoring and blood sample collection. A three-lead surface ECG was connected to the hemodynamic monitoring display (SC9000, Siemens, Germany). The animals underwent endotracheal intubation following induction of anesthesia with an intravenous injection of $3 \mu \mathrm{g} / \mathrm{kg}$ sufentanil, $1 \mathrm{mg} / \mathrm{kg}$ propofol, and $0.5 \mathrm{mg} / \mathrm{kg}$ of rocuronium. A central venous access for drug infusion was obtained via a three-lumens central venous line inserted into the right external jugular vein (Edwards LifeSciences ${ }^{(}$, California, USA). General anesthesia and analgesia were achieved using continuous inhalation of 1.8 to $2.5 \%$ sevoflurane of minimal alveolar concentration (MAC) and continuous infusion of sufentanil 1 to $4 \mu \mathrm{g} / \mathrm{kg} / \mathrm{h}$, adapted according to the response to painful stimulations, in association with 1 to $2 \mathrm{mg} / \mathrm{kg} / \mathrm{h}$ rocuronium continuous infusion to avoid shivering.

Sevoflurane is the most popular volatile agent used to induce general anesthesia, thanks to its safety profile $(20,21)$ : it has low myocardial depressant effect (22); it does not alter the A$\mathrm{H}$ interval, His-Purkinje conduction time ( $\mathrm{H}-\mathrm{V}$ interval), and ventricular conduction time (H-S interval) (23). It is associated with higher hemodynamic stability and fewer arrhythmic events compared to other volatile agents (24). Since it has no effect on the cardiac conduction system, sevoflurane can also be used in cardiac electrophysiological procedure (25). Additionally, at clinical concentrations of this drug, despite the reduction of peripheral vascular resistance, the cardiac output is preserved $(26,27)$, as well as coronary blood flow (21).

Mechanical ventilation was performed in a volume-controlled mode (Primus ${ }^{\circledR}$, Draëger, Germany) with tidal volume of $8 \mathrm{ml} / \mathrm{kg}$ and a positive end-expiratory pressure set at $5 \mathrm{~cm} \mathrm{H}_{2} \mathrm{O}$.

A 7 Fr introducer was inserted into the left external jugular vein, and a pulmonary artery catheter (CCO; Edwards LifeSciences $\left.{ }^{(}\right)$, California, USA) was advanced in a pulmonary artery for continuous cardiac output (CO), right heart pressures, and mixed venous oxygen saturation $\left(\mathrm{SVO}_{2}\right)$ monitoring. A 5 Fr introducer (Terumo Corporation, Japan) was inserted into the right internal carotid artery, and a coronary guide catheter (Sherpa JL4 ${ }^{\mathrm{TM}}$, Medtronic, Belgium) was positioned into the left coronary ostium under fluoroscopic guidance with iodinate contrast media angiogram (Xenetix $350^{\circledR}$, Guerbet, France). Through this latter step and after an intracoronary bolus of $200 \mu \mathrm{g}$ dinitrate isosorbide to prevent coronary spasm, pressure and a Doppler flow wires (ComboWire ${ }^{\circledR}$, Volcano Corporation, Belgium) were placed distally into the mid LAD. Two $5 \mathrm{Fr}$ introducers (Terumo Corporation, Japan) were inserted into the left carotid artery and left femoral artery, where high-fidelity left ventricular pressure-volume catheter (Transonic ${ }^{\circledR}$, France) and aortic arch catheter (Transonic ${ }^{\circledR}$, France) were placed.

ECG, pressure and volume signal, $\mathrm{CO}$, and respiratory rate were recorded using a data acquisition software (Notocord$\mathrm{HEM}^{\mathrm{TM}}$, France), allowing subsequent offline analysis. The animals were administrated with $300 \mathrm{mg}$ amiodarone, followed by continuous infusions of $900 \mathrm{mg} / 24 \mathrm{~h}$ and 7,500 units of unfractionated heparin, followed by a continuous infusion of 2,000 units/h.

A semi-compliant angioplasty balloon (Trek, Abbott, Belgium) was inserted over the wire into the proximal LAD and was inflated to reduce coronary flow by $60 \%$ of the baseline value for $90 \mathrm{~min}$. After $90 \mathrm{~min}$ of ischemia, $200 \mathrm{mg}$ of aspirin was administrated intravenously, and the balloon was deflated, allowing reperfusion to occur according to the best current clinical managing of ACS (28). Once the balloon was deflated, the effectiveness of reperfusion was confirmed by the recovery of intracoronary blood flow velocity. Three swine died, during the procedure, from refractory ventricular arrythmias, which occurred within the first $30 \mathrm{~min}$ from coronary occlusion.

\section{Sham Group}

A sham group of another experimental procedure (Ethical Committee acceptance number: $641 \mathrm{~N}$ ), following the same protocol of general anesthesia and instrumentation of the animal, was used as a reference to rule out the possible depressant effect of general anesthesia on the hemodynamic parameters. This sham group was composed of three crossbreed Landrace/Large White adult swine (weight: 41, 31, and $46 \mathrm{~kg}$ ), all undergoing the same general anesthesia protocol and instrumentation that we used in the present investigation. The hemodynamic parameters of each animal were followed at three different timepoints: during BSL, at $2 \mathrm{~h}$ (T1), and at $4 \mathrm{~h}$ (T2) of steady state, while no intervention was realized. These data show a reduction by $\pm 5 \mathrm{mmHg}$ of mean arterial pressure, concomitant to the experimental setting 
(Supplementary Table 1). Additionally, no arrhythmic events were observed.

Since the results from a sham group were already available in our laboratory, the local ethical committee for animal care considered it unnecessary to add a sham group in the present investigation.

\section{Accelerometric Signal Acquisition and Processing}

The KCG consisted of two modules, each containing MEMS accelerometers and gyroscope sensors (LSM6DSL, STMicroelectronics). One module was placed over the sternum to record local precordial vibrations (SCG); the other one was placed immediately below and externally to the left iliac crest to record one-lead ECG signal. The device was controlled remotely with a tablet connected via Bluetooth and collected a one-lead ECG and a linear (Lin) and a rotational (Rot) three-axes SCG. Details about this methodology have been described previously $(2,12)$. Observations from unpublished results demonstrate that SCG measurements are reliable and reproducible using different sensors and that the metrics of linear and rotational $i \mathrm{~K}$ are comparable.

Assuming that the cardiovascular system equates a Newtonian system, scalar metrics can be obtained from velocity and acceleration signals measured with the SCG in the linear and rotational dimensions and transmitted to the body surface as vibratory phenomena. The height and weight of the animal are used to assess inertial parameters. Knowing the acceleration of an object with a given mass $m$ and the vector force $(\vec{F})$, the kinetic energy $(K)$ can be calculated according to Equations (1) and (2) for the linear components and to Equations (3) and (4) for the rotational components.

$$
\begin{aligned}
\vec{F}(t) & =m \vec{a}(t) \\
K_{\text {Lin }}(t) & =\frac{1}{2} m\left(v_{x}^{2}(t)+v_{y}^{2}(t)+v_{z}^{2}(t)\right)
\end{aligned}
$$

where $m$ is the mass of the object, $K_{\mathrm{Lin}}$ is the linear kinetic energy, $v_{x}, v_{y}$, and $v_{z}$ are components of the measured velocity vector $\vec{v}$, and $\vec{F}$ is the force vector.

For the rotational components, the scalar metrics are calculated according to Equations (3) and (4).

$$
\begin{aligned}
\vec{\tau}(t) & =I \cdot \vec{\alpha}(t) \\
K_{\text {Rot }}(t) & =\frac{1}{2}\left(I_{x x} \omega_{x}^{2}(t)+I_{y y} \omega_{y}^{2}(t)+I_{z z} \omega_{z z}^{2}(t)\right)
\end{aligned}
$$

where $\vec{\tau}$ is the torque of force, $I$ is the momentum of inertia of the object, $\vec{\alpha}$ is the angular acceleration, $K_{\text {Rot }}$ is the rotational kinetic energy, $I_{x x}, I_{y y}$, and $I_{z z}$ are the orthogonal components of the momentum of inertia $I$ of the object, and $\omega_{x}, \omega_{y}$, and $\omega_{z}$ are the components of the measured angular velocity $\vec{\omega}$.
The time integral of $K_{\mathrm{Lin}}$ and $K_{\text {Rot }}$ over the cardiac cycle (CC) was computed for the SCG as in Equations (5) and (6).

$$
\begin{aligned}
i K_{\text {Lin }} & =\int_{C C} K_{\operatorname{Lin}}(t) \cdot d t . \\
i K_{\text {Rot }} & =\int_{C C} K_{\text {Rot }}(t) \cdot d t .
\end{aligned}
$$

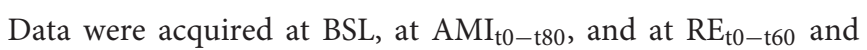
then exported and analyzed offline using a toolbox developed in MatLab version $9.5 \mathrm{R} 2018 \mathrm{~b}$ (Mathworks ${ }^{\circledR}$ ). The operator was selecting a 60-s-width artefact-free temporal window of consecutive beats. The beats were automatically identified based on the automatic identification of the peak ECG-R wave. Ensemble averaging (EA) on all beats over the selected time period was performed, and the scalar parameters of $i K_{L i n}$ and $i K_{\text {Rot }}$ were automatically computed. This method of sampling and averaging generated an averaged SCG signal which best fits the shape of a cardiac cycle. Additionally, EA was used to partially remove motion artifacts from the signals.

The P, Q, R, S, and T waves on the ECG were automatically identified and used as reference points for the identification of the electrical cardiac cycle. The sum of QRS and ST segments identifies the systolic phase (Sys) of the cardiac cycle; the sum of the TP' segment (the period from the $\mathrm{T}$ wave of the current beat $N$ to the $\mathrm{P}$ wave of the next beat $N+1$ ) with the $\mathrm{P}^{\prime} \mathrm{Q}$ ' segment (the period from the $\mathrm{P}$ wave of the beat $N+1$ to the $\mathrm{Q}$ wave of the beat $N+1$ ) identifies the diastole (Dia) of the cardiac cycle. The sum of PQ, QRS, ST, and TP' defined a whole CC. One record had to be ruled out from final analysis because of technical failure during the signal processing.

Several factors can contaminate the BCG and SCG signals, such as respiration, involuntary movements, and cough. To reduce contamination signals from artifacts, an automatic outlier detection was applied on beats that would generate too large energies, possibly due to the involuntary movement of the subject such as coughing or deglutition or movements of the extremities. If the $i \mathrm{~K}$ of a heartbeat was higher than five times the median of the respective kinetic energy of the five previous beats, the $i \mathrm{~K}$ of the concerned heartbeat was considered as compromised by a motion artefact and classified as abnormal.

Respiration might influence the BCG and SCG signals in three different ways: by producing a wandering of the baseline as a result of chest movement, by modifying the amplitude of SCG due to intra-thoracic pressure variation, and through the induced RR interval changes during the respiratory cycle. To avoid contamination signal from respiratory movement, a high-pass filter was applied to the signals.

\section{Statistical Analysis}

Statistical analysis was performed using STATACorp ${ }^{\circledR}$ for Windows. GraphPad PRISM ${ }^{\circledR}$ version 5.01 and MatLab version 9.5 R2018b (Mathworks Inc. ${ }^{\circledR}$ ) were used for graphing figures on Windows.

Normality of data distribution was assessed graphically and by using the Kolmogorov-Smirnov test. According to the distribution, data were expressed as mean \pm standard deviation 
$( \pm \mathrm{SD})$ if normally distributed or as median and interquartile range if not $\left[P_{25}-P_{75}\right]$.

To evaluate the effect of AMI and reperfusion on SCG signals, a generalized mixed model was used, taking time as the fixed factor, followed by multiple comparison whenever a significant effect was found. Bonferroni's correction was applied to account for multiple comparisons.

The pulse pressures of LV, aortic, femoral, and pulmonary artery pressures were calculated as the difference between systolic and diastolic pressures (29). Generalized linear mixed model was used to associate the pulse pressures and $\mathrm{CO}$ with the parameters of $i K$.

Spearman's rank correlation was used to assess the association of $i K$ parameters with $\Delta$ troponins and the LVEF. Correlations were calculated between $i \mathrm{~K}$ parameters and LVEF computed at the end of the procedure $\left(\mathrm{RE}_{\mathrm{t} 60}\right)$.

$P$-values $<0.05$ were considered as statistically significant.

\section{RESULTS}

Upon LAD sub-occlusion, 77\% (thirteen out of 17) of STEMI and $24 \%$ (four out of 17) of NSTEMI were observed.

Compared to baseline, troponins increased from 13.0 (6.5; $21.3) \mathrm{ng} / \mathrm{dl}$ to $170.5(102.5 ; 475.0) \mathrm{ng} / \mathrm{dl}$ and LVEF dropped from 65.0 to $30.6 \pm 5.7 \%$ at the end of revascularization (both $p<$ 0.0001). Regional LV wall abnormalities were observed as follows: anterior MI, $17.6 \%$ (three out of 17); septal MI, 5.8\% (one out of 17 ); antero-septal MI, $47.1 \%$ (eight out of 17); and inferoseptal, $29.4 \%$ (five out of 17). The animals did not disclose valve diseases at baseline, and there were no AMI-related valve diseases throughout the study.

Modifications of heart rate (HR), CO, systolic and diastolic LV pressures (PLV Sys and PLV Dia, respectively), systolic and diastolic aortic pressures (PAo Sys and PAo Dia, respectively), systolic and diastolic femoral pressures (Pfem Sys and Pfem Dia, respectively), and systolic and diastolic pulmonary artery pressures (PAP Sys and PAP Dia, respectively) during LAD sub-occlusion and reperfusion are reported in Table $\mathbf{1}$.
Figure 1 reports the modifications of pulse pressures of the same hemodynamic variables: LV pulse pressure (LV PP), aortic pulse pressure (Ao PP), femoral pulse pressure (Fem PP), and pulmonary artery pulse pressure (PA PP). The results are presented also in Supplementary Table 2.

HR increased by $19 \%$ from baseline to the end of reperfusion $\left(P_{\text {ALL }}=0.0001\right)$, while CO, systolic PLV, systolic PAo, and systolic Pfem decreased $\left(P_{\mathrm{ALL}}=0.0005, P_{\mathrm{ALL}}=0.005, P_{\mathrm{ALL}}=0.005\right.$, and $P_{\text {ALL }}<0.0001$, respectively).

According to a multiple-comparison analysis, the $\mathrm{HR}$ increased between $\mathrm{AMI}_{\mathrm{t} 0}$ and $\mathrm{RE}_{\mathrm{t} 60}(p=0.02)$; $\mathrm{CO}$ dropped from 5.5 to $4.7 \mathrm{l} / \mathrm{min}$ from BSL to $\mathrm{AMI}_{\mathrm{t} 80}$ and $\mathrm{RE}_{\mathrm{t} 0}$ (both $p=0.002$ ) and dropped further to $4.4 \mathrm{l} / \mathrm{min}$ at $\mathrm{RE}_{\mathrm{t} 60}$ compared to BSL ( $p<$ $0.0001)$; the systolic pressures of LV and aorta both dropped by 16 and $19 \%$, respectively; between BSL and $\mathrm{AMI}_{\mathrm{t} 0}(p=0.01$ and $p=0.03$, respectively) by 16 and $26 \%$, respectively; between BSL and $\mathrm{AMI}_{\mathrm{t} 80}(p=0.002$ and $p=0.003$, respectively) by 16 and $15 \%$, respectively, between BSL and $\mathrm{RE}_{\mathrm{t} 0}(p=0.001, p=0.002$, respectively), and by 16 and $15 \%$ between $\mathrm{BSL}$ and $\mathrm{RE}_{\mathrm{t} 60}(p=$ 0.003 and $p=0.036$, respectively). The systolic femoral pressure dropped between BSL and $\mathrm{AMI}_{\mathrm{t} 0}(18 \%), \mathrm{AMI}_{\mathrm{t} 80}(19 \%)$, and $\mathrm{RE}_{\mathrm{t} 0}$ (15\%) $(p<0.0001, p=0.004$, and $p<0.0001$, respectively).

When considering the pulse pressures of the above mentioned hemodynamic variables shown in Table 1, LV PP, Ao PP, and Fem PP decreased by 13, 20, and $21 \%$ from baseline to the end of reperfusion, respectively $\left(P_{\mathrm{ALL}}=0.0007, P_{\mathrm{ALL}}<0.0001\right.$, and $P_{\text {ALL }}<0.0001$, respectively). According to a multiplecomparison analysis, the Ao PP and the Fem PP dropped at $\mathrm{AMI}_{\mathrm{t} 0}$ (both $p=0.01$ ), at $\mathrm{AMI}_{\mathrm{t} 80}(p<0.0001$ and $p=$ 0.002 , respectively), and at $\mathrm{RE}_{\mathrm{t} 0}$ (both $p<0.0001$ ) compared to BSL. The modifications of pulse pressures during the LAD sub-occlusion and reperfusion are shown in Figure $\mathbf{1}$ and are reported in Supplementary Table 3.

Figure 2 depicts the modifications of parameters of $i K$ in the linear and rotational dimensions during the procedure.

All parameters of $i K$, except $i K_{R o t}^{D i a}$, decreased during LAD sub-occlusion and reperfusion. In the linear dimension, $i K_{L i n}^{C C}$, $i K_{L i n}^{\text {Sys }}$, and $i K_{L i n}^{D i a}$ dropped by 43,52 , and $53 \%$, respectively $\left(P_{\mathrm{ALL}}\right.$ $<0.0001, P_{\mathrm{ALL}}<0.0001$, and $P_{\mathrm{ALL}}=0.03$, respectively) from

TABLE 1 | Modification of hemodynamic parameters during left anterior descending occlusion and reperfusion.

\begin{tabular}{|c|c|c|c|c|c|c|c|c|c|c|}
\hline Time & HR (bpm) & CO (L/min) & $\begin{array}{l}\text { PLV Sys } \\
\text { (mmHg) }\end{array}$ & $\begin{array}{l}\text { PLV Dia } \\
(\mathrm{mmHg})\end{array}$ & $\begin{array}{l}\text { PAo Sys } \\
\text { (mmHg) }\end{array}$ & $\begin{array}{l}\text { PAo Dia } \\
\text { (mmHg) }\end{array}$ & $\begin{array}{c}\text { Pfem Sys } \\
\text { (mmHg) }\end{array}$ & $\begin{array}{c}\text { Pfem Dia } \\
\text { (mmHg) }\end{array}$ & $\begin{array}{l}\text { PAP Sys } \\
(\mathrm{mmHg})\end{array}$ & $\begin{array}{l}\text { PAP Dia } \\
(\mathrm{mmHg})\end{array}$ \\
\hline BSL & $74 \pm 14$ & $5.3 \pm 1.1$ & $96 \pm 8$ & $3 \pm 6$ & $94 \pm 6$ & $55 \pm 6$ & $98 \pm 20$ & $52 \pm 14$ & $36 \pm 5$ & $16 \pm 4$ \\
\hline $\mathrm{AMI}_{\text {to }}$ & $77 \pm 14$ & $5.1 \pm 0.8$ & $80 \pm 9^{\star}$ & $5 \pm 4$ & $78 \pm 10^{\star}$ & $47 \pm 10$ & $80 \pm 17^{\ddagger}$ & $47 \pm 14$ & $29 \pm 6$ & $14 \pm 4$ \\
\hline $\mathrm{AMI}_{\mathrm{t} 80}$ & $85 \pm 18$ & $4.7 \pm 0.85^{\dagger}$ & $80 \pm 6^{\star}$ & $5 \pm 4$ & $79 \pm 7^{\star}$ & $50 \pm 8$ & $79 \pm 22^{\star}$ & $46 \pm 16$ & $33 \pm 4$ & $16 \pm 3$ \\
\hline$R E_{t 0}$ & $87 \pm 18$ & $4.7 \pm 0.64^{\dagger}$ & $81 \pm 7^{\star}$ & $5 \pm 4$ & $80 \pm 8^{\star}$ & $49 \pm 9$ & $83 \pm 22^{\ddagger}$ & $47 \pm 17$ & $33 \pm 4$ & $16 \pm 4$ \\
\hline $\mathrm{RE}_{\mathrm{t} 60}$ & $89 \pm 17^{\star a}$ & $4.4 \pm 0.96^{\ddagger}$ & $82 \pm 7^{\star}$ & $5 \pm 3$ & $80 \pm 9^{*}$ & $49 \pm 11$ & $86 \pm 18$ & $50 \pm 11$ & $33 \pm 11$ & $15 \pm 5$ \\
\hline PALL value & 0.0001 & 0.0001 & 0.005 & ns & 0.005 & ns & 0.0001 & ns & ns & ns \\
\hline
\end{tabular}

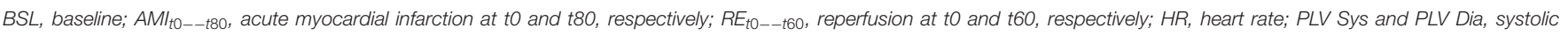

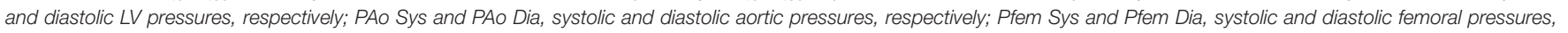
respectively; PAP Sys and PAP Dia, systolic and diastolic pulmonary arterial pressures, respectively.

Results from multiple-comparison analysis account for comparison of the different timepoints against BSL. Data are presented as mean $\pm S D$.

${ }^{*} p<0.05 ;{ }^{\dagger} p<0.01 ;{ }^{\ddagger} p<0.0001$.

${ }^{a}$ Comparison is significant against AMltO. 


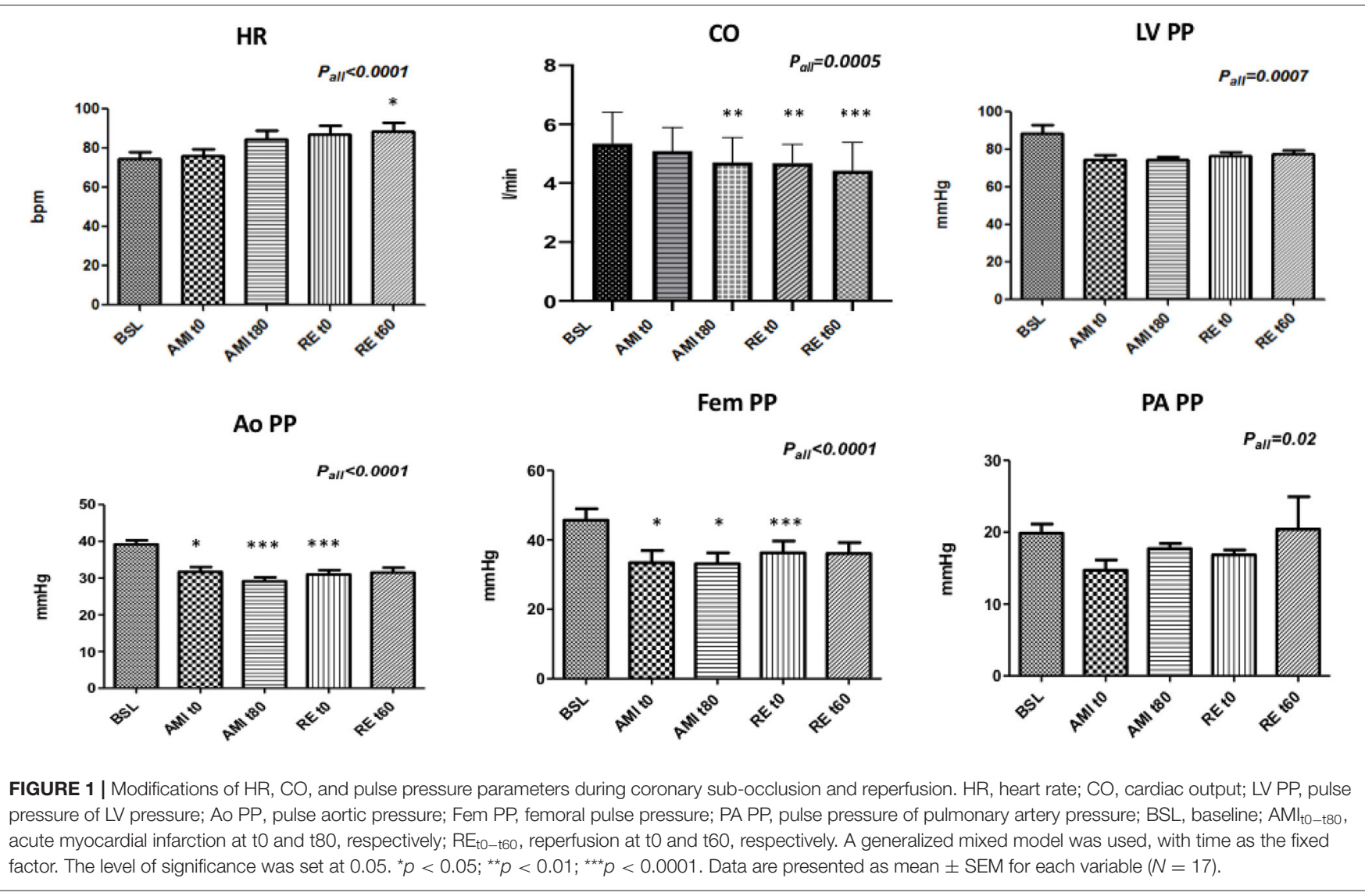

baseline to the end of reperfusion. In the rotational dimension, $i K_{R o t}^{C C}$ and $i K_{R o t}^{S y s}$ dropped by 30 and $38 \%$, respectively $\left(P_{\mathrm{ALL}}=\right.$ 0.0006 and $P_{\mathrm{ALL}}<0.0001$, respectively).

According to multiple comparisons, $i K_{\text {Lin }}^{C C}$ dropped by 20 , 30 , and $43 \%$ at $\mathrm{AMI}_{\mathrm{t} 0}, \mathrm{RE}_{\mathrm{t} 0}$, and $\mathrm{RE}_{\mathrm{t} 60}$, respectively, compared to $\operatorname{BSL}(p=0.01, p=0.007$, and $p=0.0009$, respectively); $i K_{\text {Lin }}^{\text {Sys }}$ dropped by 33,45 , and $52 \%$ at $\mathrm{AMI}_{\mathrm{t} 0}, \mathrm{RE}_{\mathrm{t} 0}$, and $\mathrm{RE}_{\mathrm{t} 60}$, respectively, compared to BSL ( $p=0.003, p=0.008$, and $p=$ 0.002 , respectively); $i K_{\text {Lin }}^{\text {Dia }}$ dropped by $53 \%$ from BSL to $\mathrm{RE}_{\mathrm{t} 60}$ $(p=0.005)$. With regards to the rotational parameters of $i \mathrm{~K}$, $i K_{\text {Rot }}^{C C}$ dropped by 20,30 , and $30 \%$ at $\mathrm{AMI}_{\mathrm{t} 0}, \mathrm{RE}_{\mathrm{t} 0}$, and $\mathrm{RE}_{\mathrm{t} 60}$, respectively, compared to BSL ( $p=0.01, p=0.01$, and $p=0.003$, respectively); $i K_{\text {Rot }}^{\text {Sys }}$ dropped by 25,38 , and $38 \%$ from BSL to $\mathrm{AMI}_{\mathrm{t} 80}, \mathrm{RE}_{\mathrm{t} 0}$, and $\mathrm{RE}_{\mathrm{t} 60}$, respectively $(p=0.008, p=0.003$, and $p<0.0001$, respectively).

Figure 3 shows a representative case of modifications of $i K$ during coronary occlusion and reperfusion for one animal.

Table 2 shows the generalized linear model used to associate pulse pressure parameters and $\mathrm{CO}$ with parameters of $i K$. All of the hemodynamic parameters, except PA PP, were significantly related to the parameters of $i K$, with a positive direction of association. LV PP was positively associated with $i K_{\text {Lin }}^{C C}, i K_{\text {Lin }}^{\text {Sys }}$, $i K_{\text {Lin }}^{D i a}, i K_{\text {Rot }}^{C C}$, and $i K_{\text {Rot }}^{\text {Sys }}(p<0.0001, p<0.0001, p=0.03, p$ $<0.0001$, and $p<0.0001$, respectively); $i K_{\text {Lin }}^{C C}, i K_{\text {Lin }}^{S y s}, i K_{R o t}^{C C}$, and $i K_{\text {Rot }}^{\text {Sys }}$ were positively associated with the Ao PP $(p<0.001, p$ $<0.001, p=0.008$, and $p=0.001$, respectively) and Fem PP ( $p=0.01, p=0.005, p=0.05$, and $p=0.01$, respectively). Although these associations were positive and significant, they were still indirect, as shown by the too wide confidence intervals. The $\mathrm{CO}$ was also found to correlate with parameters of $i \mathrm{~K}$, especially with $i K_{L i n}^{C C}, i K_{L i n}^{S y s}, i K_{R o t}^{C C}$, and $i K_{R o t}^{S y s}$, with a positive direction of association $(p=0.002, p<0.0001, p=0.004$, and $p<0.0001$, respectively).

The parameters of $i K$ have been associated to the $\Delta$ troponins and to the LVEF obtained at the end of the procedure $\left(\mathrm{RE}_{\mathrm{t} 60}\right)$, but no significant associations were observed (Tables 3 , 4 , respectively).

\section{DISCUSSION}

We reported for the first time the direct evidence that noninvasive, multi-dimensional SCG can quantify the cardiac kinetic energy and continuously track its changes during AMI and reperfusion in a closed chest swine model of AMI. We have previously highlighted the potential of micro-accelerations and gyroscopes in providing reliable information on the contractility status of the heart $(2,30)$ : as found in previous study, metrics of $i K$ are able to follow changes in cardiac contractility with high accuracy and were related to SV and CO (2); the increased 


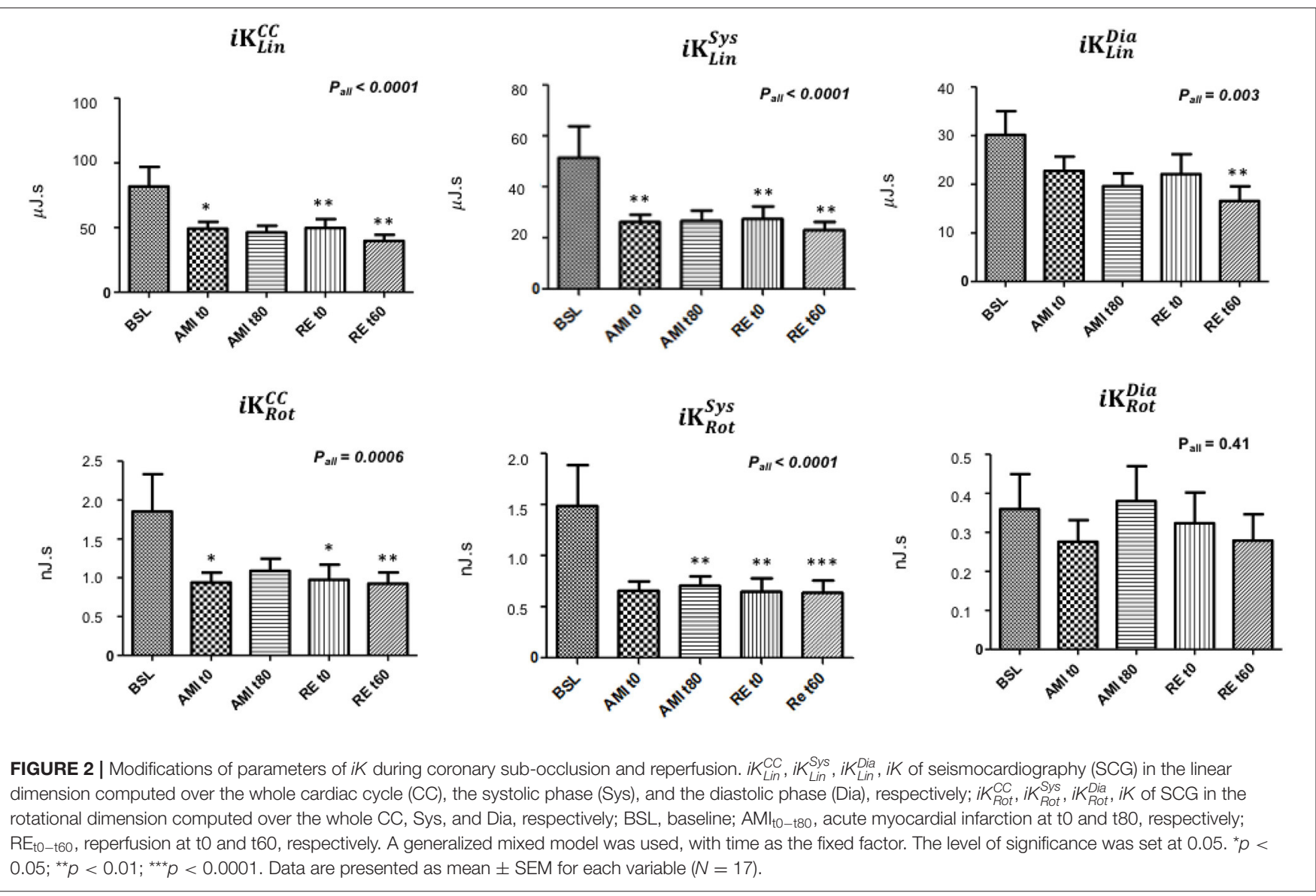

cardiac kinetic energy measured with micro-accelerometers and gyroscopes was directly related to the rise of sympathetic nerve traffic during an end-voluntary maximal apnea (31); signals acquired with multi-dimensional SCG and BCG could monitor cardiac deconditioning in astronauts during simulated microgravity (32). With the present research, we demonstrate, for the first time, that the cardiac kinetic energy recorded with multi-dimensional non-invasive SCG, along with hemodynamic and echocardiographic findings, drops during AMI compared to normal cardiac inotropic state and does not improve during coronary reperfusion (33), likely reflecting a reduced left ventricular function of ischemic origin and further confirmed by the rise of plasma troponin levels and the drop of LVEF associated with regional LV wall abnormalities, which persisted despite revascularization. After sudden coronary artery occlusion, the unsupplied myocardium loses its ability to shorten and lengthen, and myocardial contractile function drastically drops (34). With relief of ischemia and reestablishment of coronary blood flow, there is a persistent wall motion abnormality despite reperfusion and viable myocytes (35). The sudden drop of $i K$ observed immediately after coronary occlusion likely reflects the ischemic dysfunction due to supply lost, and the persistent drop of $i K$ during reperfusion likely reflects further the myocardial reperfusion injury $(33,35)$. These conclusions are further supported by the drop of LVEF and the rise of plasma troponins at the end of revascularization and further corroborated by modifications of hemodynamic parameters showing the same trend of $i \mathrm{~K}$ parameters during the experimental AMI.

Acute activation of sympathetic nervous system following AMI has been previously described in several investigations (36): the acute surge of catecholamines observed during prolonged acute myocardial ischemia (longer than $10 \mathrm{~min}$ at least) can reach plasma concentrations as high as 1,000 times of normal plasma levels, especially in cardiogenic shock (37), and such high concentrations are cardiotoxic, potentially inducing myocardial necrosis (38), with myocardial detrimental effect (37). Thus, the cardiotoxic effect of catecholamines secondary to sympathetic overactivity may be evoked as an additional mechanism contributing to the persistent drop of LVEF and $i \mathrm{~K}$ parameters after reperfusion.

Previous authors extensively investigated the utility of micro-accelerometers and gyroscopes as diagnostic tools for acute ischemic myocardial impairment (7-9), and results are all in favor to suggest the potential of micro-accelerometers and gyroscopes in the early detection of myocardial dysfunction of ischemic origin. Backer et al. used the SCG to detect myocardial impairment on nine swine and differentiate ischemia from hypovolemia as causes of myocardial dysfunction (7); Elle and colleagues used a three-axes accelerometer sensor on three anesthetized swine to recognize regional myocardial ischemia early following LAD surgical occlusion and found that the acceleration signals dropped by $40 \%$ at only $130 \mathrm{~s}$ after 


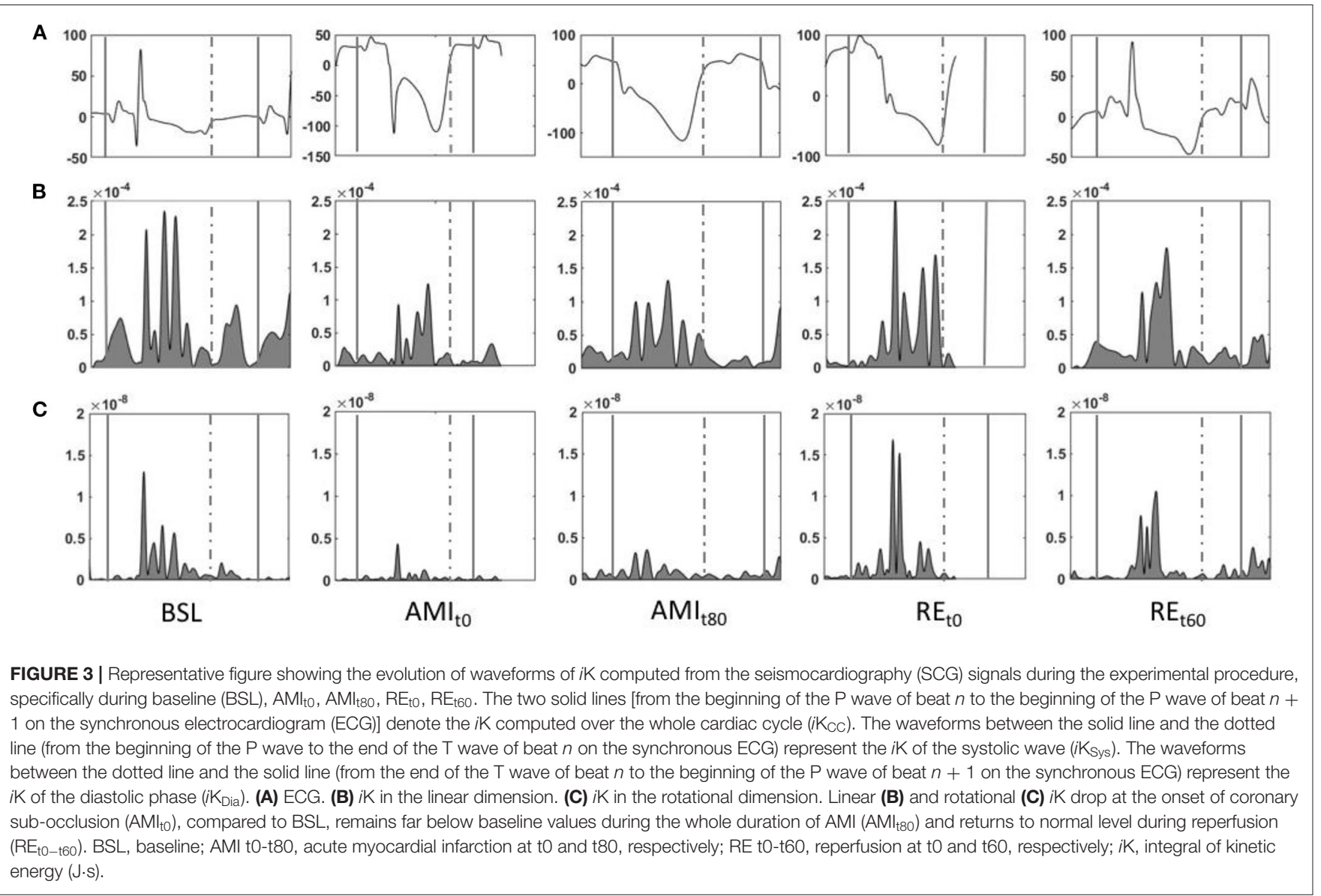

coronary occlusion; Halvorsen et al. operated on 14 anesthetized swine a LAD surgical occlusion for $60 \mathrm{~s}$ while recording the velocities of LV wall with a three-axes accelerometer and reported that myocardial wall regional impairment is accompanied by concurrent changes in accelerometer velocities both during systole and relaxation (9). They further demonstrated the potential of accelerometers in the detection of myocardial ischemia in patients undergoing cardiac surgery (13).

The present investigation strongly reinforces and complements the previous ones by adding several novelties. First, we used three-axial sensors in three cardinal axes provided with linear and rotational channels to obtain a multi-dimensional assessment of blood flow and cardiac function with six degrees of freedom. Second, we applied Newtonian equations on acceleration signals to compute the scalar parameters of kinetic energy and its temporal integral $i K$ for each contractile cycle in order to quantitatively measure the cardiac kinetic energy produced during a contractile cycle as well as during the systolic and diastolic phases $(2,11,39)$. Third, we demonstrated that the fall of cardiac $i K$ following LAD sub-occlusion is maintained for the whole duration of the coronary occlusion and does not improve during reperfusion. The fall of $i \mathrm{~K}$ parameters is likely of ischemic origin as suggested by the rise of plasma troponins and by the drop of LVEF along with regional LV wall abnormalities, which persist at the end of the experimental procedure. Fourth, changes of $i K$ parameters during the whole procedure were positively correlated with changes of invasive pulse pressures and $\mathrm{CO}$, which fell as well during acute myocardial infarction, showing the same evolution pattern of $i \mathrm{~K}$ parameters. Fifth, the drop of $i K$ observed during occlusion and reperfusion was not related to the infarct size as estimated by early troponins release nor to the severity of myocardial contraction as estimated by the LVEF. Sixth, associations between $i \mathrm{~K}$ and invasive pulse pressures are observed only with left-side pulse pressures, that is, LV PP, Ao PP, and Fem PP, but not with PA PP. Seventh, we used a closed-chest porcine model of AMI, which represents a valuable and suitable surrogate for myocardial infarction in humans (40). Reduction by $60 \%$ of coronary blood flow, induced by using a coronary balloon, was enough to trigger electrical, metabolic, and mechanical modifications of cardiac function as demonstrated by ST segment abnormalities, the rise of cardiac troponins, and the drop of LVEF along with regional LV wall abnormalities. Additionally, the sample size accounted for 17 out of 21 pigs that is far larger compared to previous investigations $(5,8,9,13)$. This observation makes the authors believe that, in this very context of experimental AMI with acute LV regional dysfunction and no concomitant AMI-related heart valve disease, linear and rotational $i \mathrm{~K}$ parameters, in particular systolic ones, provide 


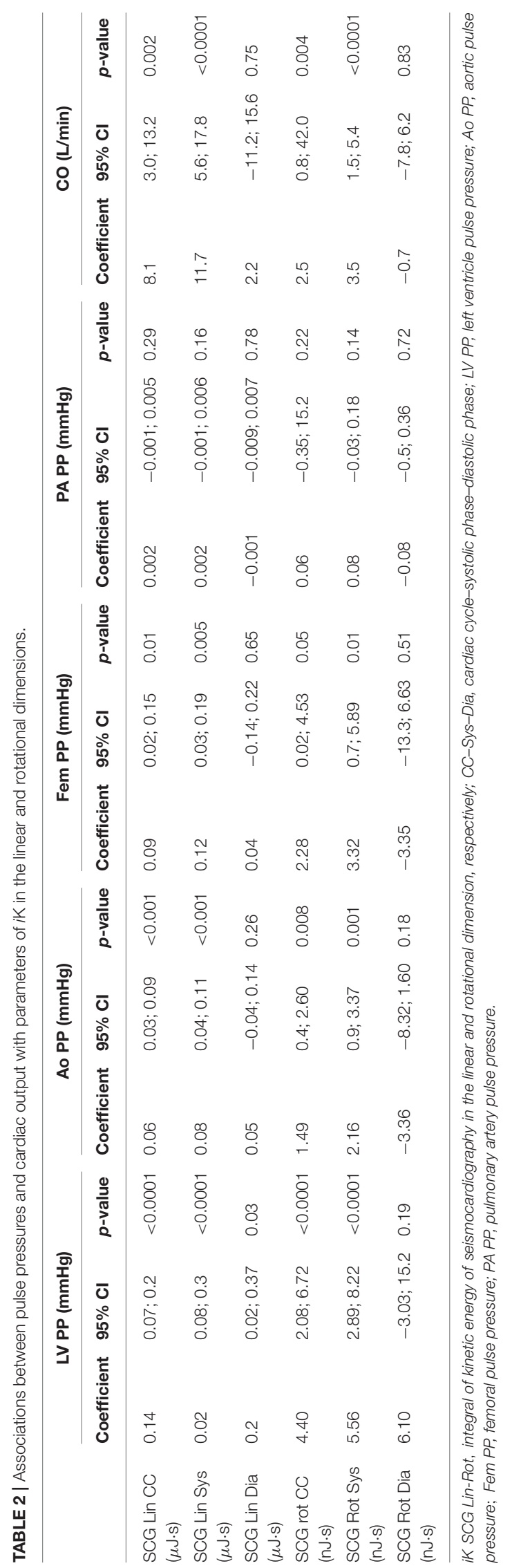

TABLE 3 | Spearman's correlation between delta troponins and parameters of $i K$.

\begin{tabular}{lll}
\hline $\boldsymbol{N}=\mathbf{1 7} \quad \boldsymbol{i} \boldsymbol{K}_{\boldsymbol{c c}}$ & $\boldsymbol{i}_{\text {Sys }}$ & $\boldsymbol{i \boldsymbol { K } _ { \boldsymbol { D i a } }}$ \\
\hline Linear dimension & & \\
$\Delta$ Troponins $r=-0.32, p=0.23$ & $r=-0.12, p=0.66$ & $r=-0.36, p=0.2$ \\
Rotational dimension & & \\
$\Delta$ Troponins $r=-0.17, p=0.53$ & $r=0.06, p=0.8$ & $r=-0.16, p=0.56$
\end{tabular}

$\Delta$ Troponins $r=-0.17, p=0.53 \quad r=0.06, p=0.8 \quad r=-0.16, p=0.56$

$i K_{C C}, i K_{S y s}$, iK Dia: iK of seismocardiography computed over the whole cardiac cycle (CC), the systolic phase (Sys), and the diastolic phase (Dia), respectively.

TABLE 4 | Spearman's correlation between LVEF and parameters of iK.

\begin{tabular}{lcc}
\hline $\boldsymbol{N}=\mathbf{1 7} \boldsymbol{i} \boldsymbol{K}_{\boldsymbol{c c}}$ & $\boldsymbol{i} \boldsymbol{K}_{\text {Sys }}$ & $\boldsymbol{i \boldsymbol { K } _ { \text { Dia } }}$ \\
\hline Linear dimension & & \\
LVEF $\quad r=-0.24, p=0.41$ & $r=-0.48, p=0.09$ & $r=0.10, p=0.74$ \\
Rotational dimension & & \\
LVEF $\quad r=-0.30, p=0.3$ & $r=-0.30, p=0.31$ & $r=-0.43, p=0.13$
\end{tabular}

$i K_{C C}, i K_{S y s}, i K_{D i a}$, iK of seismocardiography computed over the whole cardiac cycle (CC), the systolic phase (Sys), and the diastolic phase (Dia), respectively; LVEF, left ventricle ejection fraction.

reliable information on LV contractile dysfunction and its effects on the downstream circulation.

As explained above, the automatic identification of $\mathrm{P}, \mathrm{Q}$, $\mathrm{R}, \mathrm{S}$, and $\mathrm{T}$ waves on the ECG allowed for the identification of the cardiac cycle on the SCG waveforms. By combining these reference points, the systolic and diastolic phases can also be identified (12). The present investigation reported also the different impact of AMI on systolic and diastolic SCG waveforms. Indeed while the $i K$ during the systolic phase dropped during coronary sub-occlusion and reperfusion both in linear and rotational dimensions, the $i K$ during the diastolic phase seems to be less influenced by the ischemic event, showing a modest significant drop in the linear dimension and no changes at all in the rotational dimension. This makes the authors believe that an acute ischemic cardiac event with predominant systolic dysfunction has a deep impact mainly on the systolic SCG waveforms rather than the diastolic ones. The authors speculate that the diastolic component of $i \mathrm{~K}$ may reflect more the filling functions of the LV rather than its contractile properties. To further corroborate this viewpoint, the diastolic $i K$ was not associated with any of the pulse pressure parameters nor the $\mathrm{CO}$, except the linear diastolic $i \mathrm{~K}$ with the LV PP, but with a weak significance.

Even though critical differences exist between our technique and those used by our predecessors $(7-9,13,14)$ (i.e., non-intrusive device, remotely controlled system, automatic analysis, use of linear and rotational channels, computation of scalar parameters from acceleration signals), our observations further confirm the core concept that micro-accelerometers and gyroscopes can reliably monitor cardiovascular changes occurring during AMI and reperfusion.

Spaccarotella et al. have recently demonstrated that smartwatches ECG can detect ST segment elevation and 
depression with high sensitivity and specificity compared to a standard 12-lead ECG, and this might empower the earlier detection of ECG abnormalities in patients with acute coronary syndrome (41).

We presented a device capable of computing the integral of kinetic energy of a contractile cycle recorded with microaccelerometers and gyroscopes, with the aim to provide information on the mechanical activity of the heart. We previously demonstrated the capability of this device to follow changes of cardiac contractility in different conditions as already mentioned $(2,12,31,32,42)$. With the present investigation, we add that this device can detect an acutely failing heart of ischemic origin by providing a parameter of kinetic energy. An important possible application of this renewed technology is the follow-up of patients with myocardial dysfunction in the midlong term after an acute ischemic event. Thanks to the easy-to-use properties of the device, cardiac patients might be empowered to follow their own medical conditions, as it is already the case with atrial fibrillation diagnosed with smartwatches. To our knowledge, markers of myocardial mechanical function are not provided by the smartwatches currently in use, and we believe that this device may complement the existing ones by adding the cardiac kinetic energy as a new parameter of myocardial mechanical function and thus may prove useful to track changes in myocardial mechanical activity of heart failure patients in the near future.

Of course, this device must not be considered as a competitor to traditional standards and guidelines universally used for cardiac patient's follow-up but as a complement to them.

\section{Limitations}

Some limitations need considerations. Because of marked differences in anatomy, heart, and vessel orientation, the effects of myocardial infraction in humans are likely to differ in the three axes investigated in this study, but the observations on the $i K$ parameters which include the three axes should remain valid. We also cannot report on the effects of AMI on multidimensional BCG in this study because of marked differences in body mass distribution between the experimental animal model that we investigated and the human beings to which the original prototype was made for $(2,11)$.

Indeed because of technical limitations during the experimental procedure, mainly the recumbent position of the animal, the BCG sensor was placed externally to the left iliac crest and not close to the body center of mass (lower back of the animal) as recommended $(2,11)$. Placing the BCG module in this wrong position means that the recorded signals cannot be considered as BCG ones. The authors were not able to place this module over the lumbar lordosis curve for the following reasons: the recumbent position of the animal and the consequent difficulties to place the device under it and the difficulty to access this region and to promptly remove the device whenever a cardiac arrest for ventricular arrythmias occurred and prompt defibrillation was required. Indeed whenever resuscitation was required, the device was promptly removed, and easy accessibility to it was mandatory for the sake of the safety of the operators and the animal.

Despite the fact that this technique has not been standardized yet with large-scale-based studies so that no normal values of kinetic energy can be provided, this limitation was encompassed with the repeated-measures study design, where each animal was its own control. The same study design was adopted in our previous works $(2,12,31,32,42)$.

With regard to the experimental procedure, some readers may arise concerns that the observed cardiovascular modifications might be due to general anesthesia, specifically to sevoflurane (43) and azaperone (44). However, the authors are confident to conclude that the cardiovascular modifications occurring during the experimental procedure were likely attributable to acute myocardial ischemia and not to general anesthesia for several reasons: first, the drop by $\pm 5 \mathrm{mmHg}$ of the mean arterial pressure observed in the sham group cannot explain the large reduction in the mean systemic blood pressure that we observed during myocardial infarction, and this allowed the authors to rule out the depressant effect of general anesthesia on the hemodynamic parameters; second, sevoflurane has higher hemodynamic stability and fewer arrhythmic events compared to other volatile agents (24), and sevoflurane inhalation was within normal range ( 1.8 to $2.5 \%$ of MAC); third, since azaperone has a duration of action of 2 to $3 \mathrm{~h}$ in young pigs with a peak within the first $30 \mathrm{~min}$ (45) and since the procedure was started after $4 \mathrm{~h}$ at least of steady state, the effects of this drug on systemic circulation cannot be considered as responsible for the observed hemodynamic impairment after AMI; and fourth, the rise of troponin levels and the drop of LVEF associated with regional LV wall abnormalities which persist after reperfusion are all in favor to suggest that hemodynamic impairment and reduction of $i \mathrm{~K}$ parameters were a direct consequence of acute myocardial ischemia and not of anesthetic agents.

We did not find any change in the left ventricle diastolic pressure during the experimental procedure. The authors attribute this phenomenon to the effect of the mechanical ventilation with a positive end-expiratory pressure of $5 \mathrm{~cm} \mathrm{H}_{2} \mathrm{O}$, which induces a fall in transpulmonary flow and thus in the venous return to the LV, with the global effect of reducing the LV preload (46).

The study design was conceived to induce a cardiogenic shock. Since in swine only $25 \%$ of LV mass is supplied by the right coronary artery and $25 \%$ by the left circumflex artery, occluding these arteries would have probably not induced a cardiogenic shock. Further studies should be designed to assess the consequences of less extensive MI on SCG signals.

The observational period after reperfusion is relatively short; however, the study design was initially conceived to determine whether and how acute myocardial infarction and reperfusion affect the SCG signals and the derived parameters with no additional observational period. The positive and encouraging results obtained with this pivotal study set another step toward the validation of this renewed technique in the context of acute coronary diseases and undoubtedly justify further research on the long-term effect of MI on SCG signals. 
Despite the limitations described above, this study further reinforces the need to investigate on the utility of microaccelerations and gyroscopes in the detection of acute myocardial infarction on patients in real life and their potential as monitoring tools for the assessment of cardiovascular function following an acute ischemic cardiac event.

\section{CONCLUSIONS}

To our knowledge, this is the first study to demonstrate the potential of non-intrusive, multi-dimensional SCG to monitor in real time the functional status of cardiac muscle during AMI with predominant systolic dysfunction followed by coronary reperfusion and to provide a quantitative assessment of cardiac kinetic energy computed from acceleration signals. Thanks to its easy-to-use properties, this automatic and remotely controlled system may empower healthcare providers and patients to monitor cardiovascular status in real life and may help to remotely detect any cardiac functional abnormalities early. Of course, this device must not be considered as a competitor to traditional standards and guidelines universally used for a cardiac patient's follow-up but as a complement to them.

\section{DATA AVAILABILITY STATEMENT}

The raw data supporting the conclusions of this article will be made available by the authors, upon reasonable request.

\section{ETHICS STATEMENT}

The animal study was reviewed and approved by Institutional Ethics Committee on Animal Welfare from the Faculty of Medicine from the Université Libre de Bruxelles (ULB, Brussels, Belgium) (Acceptation Number: 654N).

\section{REFERENCES}

1. Gurev V, Tavakolian K, Constantino J, Kaminska B, Blaber AP, Trayanova NA. Mechanisms underlying isovolumic contraction and ejection peaks in seismocardiogram morphology. J Med Biol Eng. (2012) 32:103-10. doi: $10.5405 /$ jmbe. 847

2. Hossein A, Mirica DC, Rabineau J, Del Rio JI, Morra S, Gorlier $\mathrm{D}$, et al. Publisher correction: accurate detection of dobutamineinduced haemodynamic changes by kino-cardiography: a randomised double-blind placebo-controlled validation study. Sci Rep. (2020) 10:5459. doi: 10.1038/s41598-020-61864-9

3. Tavakolian K, Portacio G, Tamddondoust NR, Jahns G, Ngai B, Dumont GA, et al. Myocardial contractility: a seismocardiography approach. Conf Proc IEEE Eng Med Biol Soc. (2012) 2012:38014. doi: 10.1109/EMBC.2012.6346795

4. Inan OT, Etemadi M, Paloma A, Giovangrandi L, Kovacs GT. Non-invasive cardiac output trending during exercise recovery on a bathroom-scale-based ballistocardiograph. Physiol Meas. (2009) 30:261-74. doi: 10.1088/0967-3334/30/3/003

5. Calvo M, Bonnet JL, Le Rolle V, Lemonnier M, Yasuda S, Oosterlinck W, et al. Evaluation of Three-Dimensional Accelerometers for the Study of Left Ventricular Contractility. Computing in Cardiology Conference (CinC). Maastricht: IEEE (2018). doi: 10.22489/CinC.2018.176

6. Inan OT, Baran Pouyan M, Javaid AQ, Dowling S, Etemadi M, Dorier A, et al. Novel wearable seismocardiography and machine learning algorithms

\section{AUTHOR CONTRIBUTIONS}

PvdB and SM conceived the idea and the design of the study. LP designed the animal model of AMI and carried out the whole experimental procedure supported by FS and obtained all the invasive hemodynamic parameters data. AHo provided the technical support and designed the specific Toolbox in MathLab for the correct extrapolation of all metrics from SCG. SM had full access to all data in the present investigation, extrapolated all SCG data, and takes responsibility for the integrity of the data and the accuracy of the data analysis. AHe was responsible for the experimental procedure on the sham group and provided the results for the sham group. JRac performed statistical analysis. SM and LP drafted the manuscript. All authors revised the manuscript critically for important intellectual content, proofread, and made corrections to this manuscript.

\section{FUNDING}

This work was supported by the Fonds Erasme pour la Recherche Biomedicale, of the Erasme hospital, Université Libre de Bruxelles (SM, LP); by the Académie Royale de Médecine Belgique (SM); by the FNRS, Fond National pour la Recherche Scientifique, Fédération Wallonie Bruxelles, Belgium (SM, JRa); by the Fonds pour la Chirurgie Cardiaque (LP); by a grant from the European Space Agency and the Belgian Federal Scientific Policy Office (PRODEX PEA 4000110826) (AHo, DG, P-FM).

\section{SUPPLEMENTARY MATERIAL}

The Supplementary Material for this article can be found online at: https://www.frontiersin.org/articles/10.3389/fcvm. 2021.603319/full\#supplementary-material

can assess clinical status of heart failure patients. Circ Heart Fail. (2018) 11:e004313. doi: 10.1161/CIRCHEARTFAILURE.117.004313

7. Becker M, Roehl AB, Siekmann U, Koch A, de la Fuente M, Roissant R, et al. Simplified detection of myocardial ischemia by seismocardiography. Differentiation between causes of altered myocardial function. Herz. (2014) 39:586-92. doi: 10.1007/s00059-013-3851-x

8. Elle OJ, Halvorsen S, Gulbrandsen MG, Aurdal L, Bakken A, Samset E, et al. Early recognition of regional cardiac ischemia using a 3-axis accelerometer sensor. Physiol Meas. (2005) 26:429-40. doi: 10.1088/0967-3334/26/4/009

9. Halvorsen PS, Fleischer LA, Espinoza A, Elle OJ, Hoff L, Skulstad H, et al. Detection of myocardial ischaemia by epicardial accelerometers in the pig. $\mathrm{Br}$ J Anaesth. (2009) 102:29-37. doi: 10.1093/bja/aen331

10. Salerno DM, Zanetti JM, Poliac LC, Crow RS, Hannan PJ, Wang K, et al. Exercise Seismocardiography for detection of Coronary artery disease. Am J Noninvasi Cardiol. (1992) 6:321-30. doi: 10.1159/000470383

11. Migeotte PF, Mucci V, Delière Q, Lejeune L, van de Borne P. Multidimensional Kinetocardiography a New Approach for Wearable Cardiac Monitoring Through Body Acceleration Recordings. XIV Mediterranean Conference on Medical and Biological Engineering and Computing. 2016:1125-30. doi: 10.1007/978-3-319-32703-7_220

12. Morra S, Hossein A, Gorlier D, Rabineau J, Chaumont M, Migeotte PF, et al. Modification of the mechanical cardiac performance during end-expiratory voluntary apnea recorded with ballistocardiography and seismocardiography. Physiol Meas. (2019) 40:105005. doi: 10.1088/1361-6579/a $\mathrm{b} 4 \mathrm{a} 6 \mathrm{a}$ 
13. Halvorsen PS, Espinoza A, Fleischer LA, Elle OJ, Hoff L, Lundblad R, et al. Feasibility of a three-axis epicardial accelerometer in detecting myocardial ischemia in cardiac surgical patients. J Thorac Cardiovasc Surg. (2008) 136:1496-502. doi: 10.1016/j.jtcvs.2008.08.043

14. Theres HP, Kaiser DR, Nelson SD, Glos M, Leuthold T, Baumann G, et al. Detection of acute myocardial ischemia during percutaneous transluminal coronary angioplasty by endocardial acceleration. Pacing Clin Electrophysiol. (2004) 27:621-5. doi: 10.1111/j.1540-8159.2004.00496.x

15. Yang C, Tang S, Tavassolian N. Utilizing gyroscopes towards the automatic annotation of seismocardiograms. IEEE Sensors Journal. (2017) 17:212936. doi: 10.1109/JSEN.2017.2663420

16. Jafari Tadi M, Lehtonen E, Saraste A, Tuominen J, Koskinen J, Teras M, et al. Gyrocardiography: a new non-invasive monitoring method for the assessment of cardiac mechanics and the estimation of hemodynamic variables. Sci Rep. (2017) 7:6823. doi: 10.1038/s41598-017-07248-y

17. Jafari Tadi M, Lehtonen E, Teuho J, Koskinen J, Schultz J, Siekkinen R, et al. A computational framework for data fusion in MEMS-based cardiac and respiratory gating. Sensors (Basel). (2019) 19:19. doi: 10.3390/s19194137

18. Migeotte PF, Monfils J, Landreani F, Funtova II, Tank J, van de Borne P, et al. P439 Cardiac strength deconditioning after the 60-days head-down bed-rest assessed by heart kinetic energy wearable monitoring. Eur Heart J. (2017) 38:60. doi: 10.1093/eurheartj/ehx501.P439

19. Sengupta PP, Tajik AJ, Chandrasekaran K, Khandheria BK. Twist mechanics of the left ventricle: principles and application. JACC Cardiovasc Imaging. (2008) 1:366-76. doi: 10.1016/j.jcmg.2008.02.006

20. Brioni JD, Varughese S, Ahmed R, Bein B. A clinical review of inhalation anesthesia with sevoflurane: from early research to emerging topics. J Anesth. (2017) 31:764-78. doi: 10.1007/s00540-017-2375-6

21. De Hert S, Moerman A. Sevoflurane. F1000Res. 4:626. doi: 10.12688/f1000research.6288.1

22. Kanaya N, Kawana S, Tsuchida H, Miyamoto A, Ohshika H, Namiki A. Comparative myocardial depression of sevoflurane, isoflurane, and halothane in cultured neonatal rat ventricular myocytes. Anesth Analg. (1998) 87:10417. doi: 10.1213/00000539-199811000-00013

23. Nakaigawa Y, Akazawa S, Shimizu R, Ishii R, Yamato R. Comparison of the effects of halothane, isoflurane, and sevoflurane on atrioventricular conduction times in pentobarbital-anesthetized dogs. Anesth Analg. (1995) 81:249-53. doi: 10.1213/00000539-199508000-00007

24. Regueiro-Purrinos M, Fernandez-Vazquez F, de Prado AP, Altonaga JR, Cuellas-Ramon C, Ajenjo-Silverio JM, et al. Ventricular arrhythmias and mortality associated with isoflurane and sevoflurane in a porcine model of myocardial infarction. J Am Assoc Lab Anim Sci. (2011) 50:73-8.

25. Delgado-Herrera L, Ostroff RD, Rogers SA. Sevoflurance: approaching the ideal inhalational anesthetic. A pharmacologic, pharmacoeconomic, and clinical review. CNS Drug Rev. (2001) 7:48-120. doi: 10.1111/j.1527-3458.2001.tb00190.x

26. Eger EI. New inhaled anesthetics. Anesthesiology. (1994) 80:90622. doi: 10.1097/00000542-199404000-00024

27. Young CJ, Apfelbaum JL. Inhalational anesthetics: desflurane and sevoflurane. J Clin Anesth. (1995) 7:564-77. doi: 10.1016/0952-8180(95)00129-8

28. Ibanez B, James S, Agewall S, Antunes MJ, Bucciarelli-Ducci C, Bueno H, et al. 2017 ESC Guidelines for the management of acute myocardial infarction in patients presenting with ST-segment elevation: The Task Force for the management of acute myocardial infarction in patients presenting with STsegment elevation of the European Society of Cardiology (ESC). Eur Heart J. (2018) 39:119-77. doi: 10.1093/eurheartj/ehx393

29. Pareek M, Vaduganathan M, Biering-Sorensen T, Byrne C, Qamar A, Almarzooq Z, et al. Pulse pressure, cardiovascular events, and intensive bloodpressure lowering in the systolic blood pressure intervention trial (SPRINT). Am J Med. (2019) 132:733-9. doi: 10.1016/j.amjmed.2019.01.001

30. Morra S, Hossein A, Rabineau J, Gorlier D, Racape J, Migeotte P, et al. Assessment of left ventricular twist by 3D ballistocardiography and seismocardiography compared with 2D STI echocardiography in a context of enhanced inotropism in healthy subjects. Sci Rep. (2021) 11:683. doi: 10.1038/s41598-020-79933-4

31. Morra S, Gauthey A, Hossein A, Rabineau J, Racape J, Gorlier D, et al. Influence of sympathetic activation on myocardial contractility measured with ballistocardiography and seismocardiography during sustained endexpiratory apnea. Am J Physiol Regul Integr Comp Physiol. (2020) 319:497506. doi: 10.1152/ajpregu.00142.2020

32. Rabineau J, Hossein A, Landreani F, Haut B, Mulder E, Luchitskaya E, et al. Cardiovascular adaptation to simulated microgravity and countermeasure efficacy assessed by ballistocardiography and seismocardiography. Sci Rep. (2020) 10:17694. doi: 10.1038/s41598-02074150-5

33. Heusch G, Gersh BJ. The pathophysiology of acute myocardial infarction and strategies of protection beyond reperfusion: a continual challenge. Eur Heart J. (2017) 38:774-84. doi: 10.1093/eurheartj/ehw224

34. Bolli R. Mechanism of myocardial "stunning". Circulation. (1990) 82:72338. doi: 10.1161/01.CIR.82.3.723

35. Yellon DM, Hausenloy DJ. Myocardial reperfusion injury. $N$ Engl J Med. (2007) 357:1121-35. doi: 10.1056/NEJMra071667

36. Ciarka A, van de Borne P, Pathak A. Myocardial infarction, heart failure and sympathetic nervous system activity: new pharmacological approaches that affect neurohumoral activation. Expert Opin Investig Drugs. (2008) 17:131530. doi: 10.1517/13543784.17.9.1315

37. Benedict CR, Grahame-Smith DG. Plasma adrenaline and noradrenaline concentrations and dopamine-beta-hydroxylase activity in myocardial infarction with and without cardiogenic shock. Br Heart J. (1979) 42:21420. doi: 10.1136/hrt.42.2.214

38. Waldenstrom AP, Hjalmarson AC, Thornell L. A possible role of noradrenaline in the development of myocardial infarction: an experimental study in the isolated rat heart. Am Heart J. (1978) 95:43-51. doi: 10.1016/0002-8703(78)90395-2

39. Inan OT, Migeotte PF, Park KS, Etemadi M, Tavakolian K, Casanella $R$, et al. Ballistocardiography and seismocardiography: a review of recent advances. IEEE J Biomed Health Inform. (2015) 19:1414-27. doi: 10.1109/JBHI.2014.2361732

40. Suzuki Y, Yeung AC, Ikeno F. The representative porcine model for human cardiovascular disease. J Biomed Biotechnol. (2011) 2011:195483. doi: 10.1155/2011/195483

41. Spaccarotella CAM, Polimeni A, Migliarino S, Principe E, Curcio A, Mongiardo A, et al. Multichannel electrocardiograms obtained by a smartwatch for the diagnosis of ST-segment changes. JAMA Cardiol. (2020) 5:1176-80. doi: 10.1093/ehjci/ehaa946.3441

42. Morra S, Hossein A, Gorlier D, Rabineau J, Chaumont M, Migeotte PF, et al. Ballistocardiography and Seismocardiography detect hemodynamic changes during simulated obstructive apnea. Physiol Meas. (2020) 41:065007. doi: 10.1088/1361-6579/ab924b

43. Ebert TJ. Cardiovascular and autonomic effects of sevoflurane. Acta Anaesthesiol Belg. (1996) 47:15-21.

44. Lees P, Serrano L. Effects of azaperone on cardiovascular and respiratory functions in the horse. $\mathrm{Br} J$ Pharmacol. (1976) 56:263-9. doi: 10.1111/j.1476-5381.1976.tb07637.x

45. Mikota SK, Plumb DC. Elephant Care International: Elephant Formulary Azaperone. (2003). Available online at: http://elephantcare.org/resources/ formulary/drug-index/azaperone/

46. Duke GJ. Cardiovascular effects of mechanical ventilation. Crit Care Resusc. (1999) 1:388-99.

Conflict of Interest: P-FM, DG, and AHo declare having direct ownership of shares in Healthcare Company.

The remaining authors declare that the research was conducted in the absence of any commercial or financial relationships that could be construed as a potential conflict of interest.

Copyright (c) 2021 Morra, Pitisci, Su, Hossein, Rabineau, Racape, Gorlier, Herpain, Migeotte, Creteur and van de Borne. This is an open-access article distributed under the terms of the Creative Commons Attribution License (CC BY). The use, distribution or reproduction in other forums is permitted, provided the original author(s) and the copyright owner(s) are credited and that the original publication in this journal is cited, in accordance with accepted academic practice. No use, distribution or reproduction is permitted which does not comply with these terms. 